\title{
Nonequilibrium Properties of Berezinskii-Kosterlitz-Thouless Phase Transitions
}

\author{
C. Klöckner, ${ }^{1}$ C. Karrasch, ${ }^{1}$ and D. M. Kennes $\circledast^{2,3}$ \\ ${ }^{1}$ Technische Universität Braunschweig, Institut für Mathematische Physik, Mendelssohnstrae 3, 38106 Braunschweig, Germany \\ ${ }^{2}$ Institut für Theorie der Statistischen Physik, RWTH Aachen University and JARA-Fundamentals of Future Information Technology, \\ 52056 Aachen, Germany \\ ${ }^{3}$ Max Planck Institute for the Structure and Dynamics of Matter, Center for Free Electron Laser Science, 22761 Hamburg, Germany
}

(Received 21 March 2020; accepted 26 August 2020; published 1 October 2020)

\begin{abstract}
We employ a novel, unbiased renormalization-group approach to investigate nonequilibrium phase transitions in infinite lattice models. This allows us to address the delicate interplay of fluctuations and ordering tendencies in low dimensions out of equilibrium. We study a prototypical model for the metal to insulator transition of spinless interacting fermions coupled to electronic baths and driven out of equilibrium by a longitudinal static electric field. The closed system features a Berezinskii-KosterlitzThouless transition between a metallic and a charge-ordered phase in the equilibrium limit. We compute the nonequilibrium phase diagram and illustrate a highly nonmonotonic dependence of the phase boundary on the strength of the electric field: for small fields, the induced currents destroy the charge order, while at higher electric fields it reemerges due to many-body Wannier-Stark localization physics. Finally, we show that the current in such an interacting nonequilibrium system can counter-intuitively flow opposite to the direction of the electric field. This nonequilibrium steady state is reminiscent of an equilibrium distribution function with an effective negative temperature.
\end{abstract}

DOI: 10.1103/PhysRevLett.125.147601

Introduction.-Understanding the properties of quantum many-body systems that are continuously driven out of equilibrium is at the vanguard of contemporary condensed matter research [1]. A particularly intriguing avenue along these lines is the study of the interplay of nonequilibrium physics with emergent phenomena such as phase transitions, which are well understood in equilibrium [2]. Driving a system out of its equilibrium state in a controlled fashion in order to explore this interplay poses an experimental challenge, which is being mastered at an astonishing rate [3]. Reliable theoretical tools to connect to these experimental advances from the viewpoint of microscopic models are being complicated by the inherent complexity of treating a system in nonequilibrium. In low dimensions, thermal and quantum fluctuations are enhanced, which leads to a destabilization of many ordered phases of matter, especially those that break a continuous symmetry [4-6]. Even in equilibrium, capturing this competition of fluctuations and order is well beyond simple mean-field approaches which inherently underestimate the relevance of fluctuations and which thus artificially promote long-ranged order. This is particularly prominent in one dimension where quantum and thermal fluctuations do not allow for spontaneously broken continuous symmetries. There is a dire need to develop tools that aid an understanding of the nonequilibrium physics of low-dimensional systems beyond simple mean-field paradigms [7-13].

One of the most successful approaches to describe emergent phenomena in equilibrium is the so-called renormalization group ( $R G)$, which has been instrumental in understanding and describing phase transitions of classical as well as quantum many-body systems $[14,15]$. The key idea of the RG is to address energy scales successively (usually from high to low) in order to set up an effective low-energy theory that is easier to handle. In nonequilibrium a clear separation into high and low energies is ambiguous due to the continuous drive and as a consequence such an ansatz needs to correctly account for the microscopic details of the underlying model, which are often disregarded in an equilibrium RG procedure. In this Letter, we employ an outof-equilibrium RG scheme [16,17], which does keep the full microscopic information about high- and low-energy degrees of freedom. In contrast to prior applications, we do account for inelastic two-particle scattering terms. Our approach is thus an a priori well-suited candidate to explore the nonequilibrium realm.

We stress that in equilibrium and at low energies, the microscopic model studied in this Letter can be mapped to a continuous field theory (the so-called Sine-Gordon model). Following this route is tremendously insightful, but requires care in an out-of equilibrium situation. One can directly study the Sine-Gordon model in nonequilibrium, but since the separation into high- and low-energy degrees of freedom is ambiguous in the presence of a continuous drive, the underlying mapping (from a microscopic model to a continuum field theory) itself becomes less clear. For a quenched system (a system that is not continuously driven but for which the Hamiltonian is abruptly changed in time), recent pioneering works on 2D superfluids [18] and on 2D 
cold atoms [19] reveal Berezinskii-Kosterlitz-Thouless (BKT)-like behavior $[20,21]$ in the time evolution towards a steady state. Our study is complementary in spirit, as we (i) directly work with the microscopic model and (ii) analyze the steady state itself that emerges for continuous driving. This state is an inherently nonequilibrium one; e.g., it violates detailed balance, as we will demonstrate later. Our results can serve as a test bed to determine if and how microscopic models can be mapped to field theories in a continuously driven out-of-equilibrium setup.

On general grounds, a study of the nonequilibrium emergent behavior of quantum many-body systems needs to include the coupling to a bath. In a closed quantum system with genuine interactions and drive, heating is expected to push the system into a hot (asymptotically infinitetemperature) state where all interesting emergent phenomena are wiped out entirely. Therefore, continuously driving a system out of equilibrium necessitates another tuning knobthe strength of reservoir-system coupling. This will fundamentally affect the physics. Our RG procedure can account for both the driving and the coupling to reservoirs.

Our strategy is to start with a system in which a wellunderstood BKT phase transitions occurs in equilibrium $[20,21]$ and to then apply our novel framework to investigate the intriguing realm of nonequilibrium. We focus on a model of interacting lattice fermions which features a BKT transition from a metallic into a charge-ordered (insulating) state in equilibrium. Our RG calculations yield good agreement with this exact result as well as with mean-field predictions for the open system in limits where quantum fluctuations are irrelevant (e.g., for large interactions). This illustrates that our scheme is well suited to determine the out-of-equilibrium properties in arbitrary parameter regimes. As a key result, we find that a longitudinal static electric field has a profound influence on the steady-state, nonequilibrium phase diagram: a small field induces currents and can thus eventually suppress charge order and drive the system into a metallic state. For large fields, however, the current is blocked by many-body Wannier-Stark localization [22-24]: as particles move either up or down the lattice, they lose or gain energy from the linear potential gradient induced by the electric field and are finally reflected. The system consequently reenters an insulating, charge-ordered state. A second confounding observation is that the nonequilibrium steady state can evolve into one of effective negative temperature [25-27]. In such a state, the current flows opposite to the applied electric field $[28,29]$, which, in equilibrium, would signal the onset of an instability to phase separation of charge.

Model and method.-We consider an infinite interacting, one-dimensional metal in an static electric field:

$$
\begin{aligned}
H= & \sum_{n} t c_{n}^{\dagger} c_{n+1}+\text { H.c. }+n E c_{n}^{\dagger} c_{n} \\
& +U\left(c_{n}^{\dagger} c_{n}-\frac{1}{2}\right)\left(c_{n+1}^{\dagger} c_{n+1}-\frac{1}{2}\right)
\end{aligned}
$$

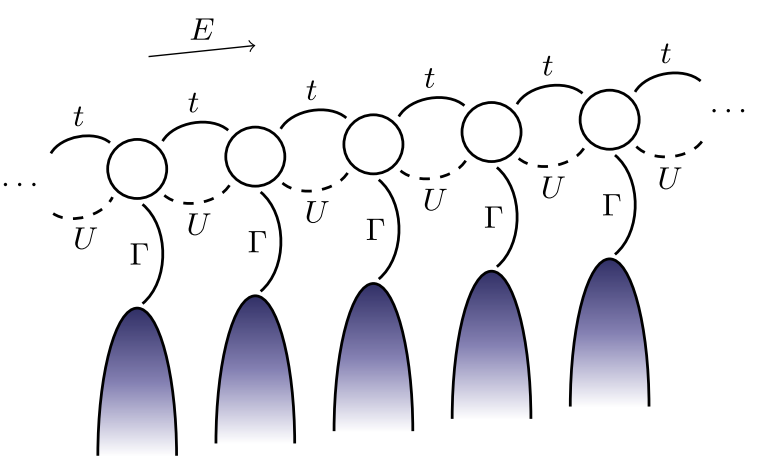

FIG. 1. The model used in this Letter: a tight-binding chain with a nearest-neighbor hopping $t$ and a nearest-neighbor interaction $U$ is coupled to electronic reservoirs via a hybridization $\Gamma$. A longitudinal electric field $E$ drives the system out of thermal equilibrium.

where $c_{n}^{(\dagger)}$ denote fermionic annihilation and creation operators. The strength of the nearest-neighbor hoppings and two-body interactions is given by $t$ and $U$, respectively. The electric field $E$ leads to an energy bias between adjacent sites. We side couple every site $n$ of this chain to an individual electronic zero-temperature $(T=0$, chemical potential $\mu_{n}=n E$ ) reservoir whose bandwidth is assumed to be large compared to all other energy scales. The reservoirs are then fully characterized by a scalar hybridization $\Gamma$. Our model is visualized in Fig. 1.

In the absence of the reservoirs and in the equilibrium limit $(\Gamma=E=0)$, the model can be solved using the Bethe ansatz [21]. The ground state is a gapless Luttinger liquid for small $U$, but charge density wave order is stabilized beyond a finite critical $U_{\text {crit }} / t=2$. The phase transition is driven by a Berezinskii-Kosterlitz-Thouless mechanism. Our goal is to determine the out-of-equilibrium phase diagram for arbitrary $\Gamma$ and $E$.

A mean-field analysis $[6,8,30]$ for $\Gamma=E=0$ yields $U_{\text {crit }}^{\mathrm{MF}} / t=0$ in stark contrast to the exact result. Critical quantum fluctuations are disregarded, and a mean-field approach is inherently insufficient to study the phase diagram. The same holds true for second order perturbation theory in $U$, which does not find a transition into a charge density wave (CDW) phase at any $U$. Thus, a more sophisticated method is required. In this Letter, we employ a novel renormalization-group framework [16] whose main ingredients are coupled flow equations for the singleparticle self-energy $\Sigma(\omega)$ as well as for the effective two-body interaction on the Keldysh contour. The fundamental approximation within this method is a truncation of the flow equations in powers of $U$. We keep all terms of $\mathcal{O}\left(U^{2}\right)$ as well as an infinite number of certain higher-order terms; we obtain exact results for $U=0$. The key advantage of our approach is that it keeps track of all microscopic details, which is essential in nonequilibrium where an a priori separation of relevant low-energy degrees of freedoms from the rest is ambiguous. In contrast to prior, 
leading-order applications for 1D systems out of equilibrium which essentially mapped the problem an effective noninteracting one [7,31,32], we incorporate inelastic processes by accounting for the energy dependence of the effective two-body interaction [33,34]. The resulting set of RG flow equations is involved, and their solution requires advanced concepts such as recursion relations for Green's functions in the presence of quasitranslation invariance. Details of this method can be found in a separate publication [35].

In contrast to both mean-field and perturbation theory, our RG approach yields a finite value of $U_{\text {crit }}$ in the limit $\Gamma=E=0$ in accord with the analytic solution [36]. Moreover, we quantitatively reproduce an exact result for the phase boundary at $\Gamma>0$ in the limit $U \rightarrow \infty$. Both are highly nontrivial tests for our method and indicate that the phase diagram can be determined reliably.

Phase diagram.-To probe spontaneous symmetry breaking and the transition into a CDW phase, we add a small staggered on site potential $s$ to the Hamiltonian [36] and compute the susceptibility

$$
\chi=\lim _{s \rightarrow 0} \frac{\left\langle\hat{n}_{\text {even }}\right\rangle-\left\langle\hat{n}_{\text {odd }}\right\rangle}{s},
$$

where $\left\langle\hat{n}_{\text {even }}\right\rangle$ and $\left\langle\hat{n}_{\text {odd }}\right\rangle$ denote the occupation of even and odd sites, respectively. For systems transitioning into a charge density wave state, $\chi$ will diverge, while it remains finite otherwise. We can thus employ $\chi$ to map out the phase diagram.

In the left and right panels of Fig. 2, we show phase diagrams in the $\Gamma-U$ plane for zero and finite electric fields, respectively. The false color plot shows the
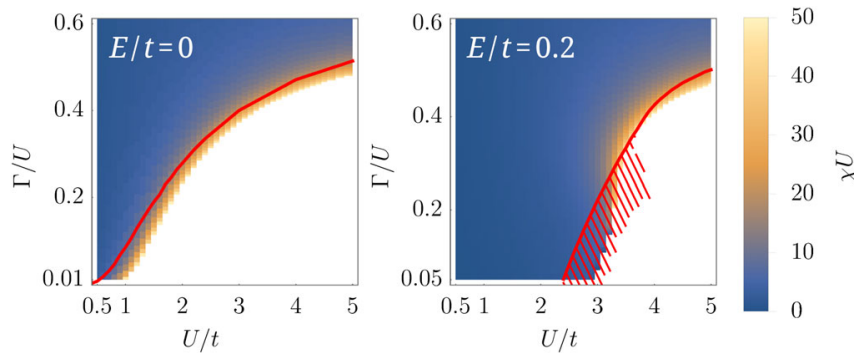

FIG. 2. Phase diagram in the $U-\Gamma$ plane for two different electric fields $E$. The color scale displays the susceptibility $\chi$ towards a charge density wave (white regions) obtained using our $\mathrm{RG}$ scheme [35]. It is known that the system is driven into a CDW phase at $U_{\text {crit }} / t=2$ in the absence of reservoirs in equilibrium $(\Gamma=E=0)$. Our RG approach yields $U_{\text {crit }}^{\mathrm{RG}} / t \approx 1.4$ in this limit in contrast to mean-field theory (thick red line), which finds $U_{\text {crit }}^{\mathrm{MF}}=0$. When a small electric field $E / t=0.2$ is switched on, currents start to flow and suppress charge order; thus, the size of the metallic phase increases. In certain areas of the phase diagram (red hatching), one finds multiple stable solutions of the meanfield equations (where at least one indicates charge order and at least one indicates a uniform state). susceptibility (2) obtained within our second-order RG scheme. The red line indicates the mean field transition into a charge-ordered state. In equilibrium $(E=0)$ and at low $\Gamma$, we find $U_{\text {crit }}^{\mathrm{RG}} / t \approx 1.4$ in accord with the exact solution and in contrast to both the mean-field $\left(U_{\text {crit }}^{\mathrm{MF}} / t=0\right)$ and perturbation theory $\left(U_{\text {crit }}^{\mathrm{PT}} / t=\infty\right)$ results. If the reservoir coupling $\Gamma$ is increased, the system is ultimately driven into a translation-invariant, disordered phase. The size of the CDW phase decreases if a small electric field is turned on: currents start to flow and tend to destroy charge order (see right panel of Fig. 2).

The mean-field equations can generally have multiple solutions, and in equilibrium the physical one can be determined by minimizing the free energy. At finite electric fields, however, it is unclear how to proceed, and it is not even guaranteed that a single stable mean-field solution exits [6]. This is explicitly illustrated in Fig. 2 where the red hatched regions show regimes where at least one solution indicating charge order and at least one stable solution indicating a uniform state can be found. Thus, it is difficult to decide the physically relevant nonequilibrium steady state using mean-field theory. Such a deficiency does not occur within our RG approach.

In Fig. 3, we investigate the influence of the electric field (which drives the system into a nonequilibrium state) in more detail and report an astonishingly delicate dependence of the phase boundary on the field strength. The three different panels show the phase diagram in the $\Gamma-E$ plane for three different values of the interaction $U$. At intermediate interaction $U / t=5$, the system is in a CDW phase for small $\Gamma$ and $E$. If the electric field is increased, currents start to flow and suppress charge order. At a large $E$, however, large site-to-site energy gradients energetically prohibit the movement of particles. In this many-body Wannier-Stark localized regime, currents vanish and the system reenters a CDW phase. For small $U / t=1$, the electric field drives the transition into a CDW phase. This illustrates the intricate nature of equilibrium BKT transitions and how their behavior can be flexibly tuned when driving the system into an out-ofequilibrium state.

If either the interaction or the electric field becomes large, we find good agreement between the mean-field prediction and our RG approach (see Fig. 3), which is reassuring as quantum fluctuations should be small in these limits. In particular, the mean-field equations can be solved analytically for $U \rightarrow \infty$ or $E \rightarrow \infty$, and a transition between a charge ordered and a metallic state occurs at a critical value of $\Gamma_{\text {crit }}^{\mathrm{MF}}=(2 / \pi) U_{\text {crit }}^{\mathrm{MF}} \approx 0.64 U_{\text {crit }}^{\mathrm{MF}}$ [35]. Our $\mathrm{RG}$ scheme yields $\Gamma_{\text {crit }}^{\mathrm{RG}} \approx 0.61 U_{\text {crit }}^{\mathrm{RG}}$. We emphasize that this represents a highly nontrivial test for our framework which is based on a truncation of a hierarchy of flow equations in powers of $U$. One should note that at large but finite $U / t=5$ and $U / t=10$, the RG approach has again a distinct advantage over the mean-field analysis: within the 


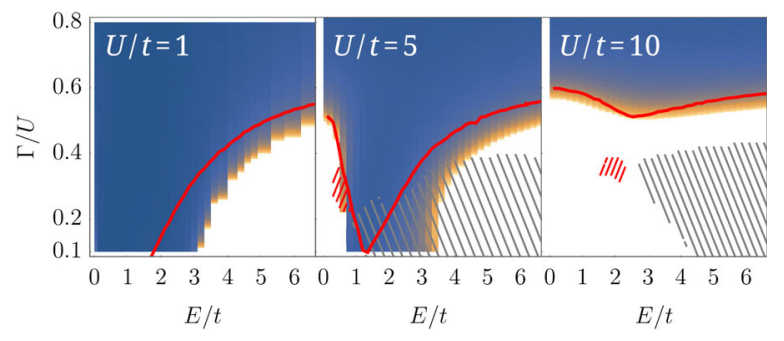

FIG. 3. Phase diagram in the $\Gamma-E$ plane for different values of $U$. At intermediate $U / t=5$ and small $\Gamma$, an electric field first induces currents and drives the system into a disordered metallic phase. For large $E$, however, many-body Wannier-Stark localization sets in and the system reenters a CDW phase. For small $U / t=1$, a large electric field can drive the transition into an ordered CDW phase. At large $U / t=10$, the phase boundary can be extracted reliably via mean-field theory (red line); our RG data is in good agreement with this prediction. Grey hatching indicates regions where a mean-field approach fails to find any stable solution. Red hatching as well as the color scale are the same as in Fig. 2.

latter, there are regions in the phase diagram where no stable solution can be obtained (grey hatching in Fig. 3).

Negative currents and temperatures.-Next, we study the metallic phase (where no charge order emerges) in more detail. We focus on the limit $\Gamma \rightarrow 0$, which is particularly interesting as correlations are expected to have the strongest effect on the nonequilibrium steady state and might lead to exotic phenomena. Figure 4(a) shows the current flowing

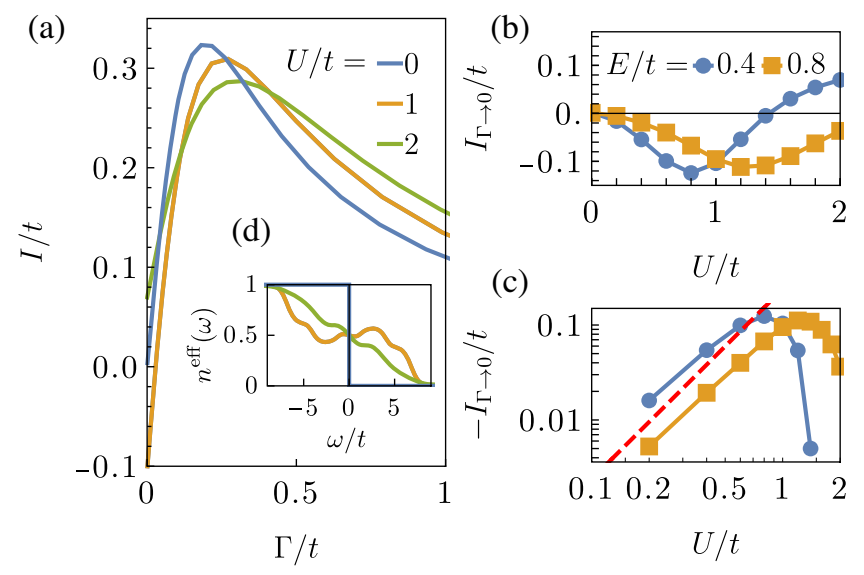

FIG. 4. The particle current induced by the electric field in the metallic phase (a) as a function of the reservoir coupling $\Gamma$ for $E / t=0.4$ and different $U$, (b),(c) as a function of the interaction $U$ in the limit $\Gamma \rightarrow 0$ for different $E$ on a linear and logarithmic scale, respectively. At finite interactions, the current does not vanish in the limit $\Gamma \rightarrow 0$ and becomes negative for sufficiently small $U$. (d) The effective distribution function (3) for $E / t=0.4$, $\Gamma \rightarrow 0$, and various $U$. At $U / t=1$, one observes a positive slope for frequencies smaller than the bandwidth. This corresponds to an effective negative temperature, which in turn leads to a (negative) current that flows opposite to the direction of the electric field. through the system as a function of the reservoir coupling $\Gamma$ for three values of the interaction. In the noninteracting case (blue line), the current $I$ first increases as $\Gamma$ is loweredparticles move with increasing ease without being hampered by the side-coupled reservoirs. At about $\Gamma / t=0.2$, the current features a maximum and decreases as $\Gamma$ is lowered further due to the onset of Wannier-Stark localization. In the presence of interactions, the maximum in the current shifts to larger values of $\Gamma / t$ and the maximal value in $I / t$ decreases. This is most likely related to the interaction-induced band broadening and to inelastic processes generated by the interaction. The most surprising effect that we report, however, is a negative current in the steady state for $\Gamma \rightarrow 0$ and small to intermediate $U$ [yellow line in Fig. 4(a)]. Only at larger $U$, the current turns positive again [green line in Fig. 4(a)]. A current that is counterpropagating to the direction of the electric field $[28,29]$ is striking and in stark contrast to a more commonly encountered negative differential conductance [37-40]. In thermal equilibrium, such a state would violate the thermodynamic stability of the system, and spontaneous charge separation would establish a new stable equilibrium configuration. However, no such constrains are present in our generic nonequilibrium setup where energy is not conserved.

It is important to demonstrate that the negative current is not an artifact-be it numerical or conceptual—of the underlying RG approach. To this end, we first check that our results are converged with respect to all of the numerical parameters (see the Supplemental Materials [41] for details). On the conceptual level, we reiterate that our method includes all terms to order $\mathcal{O}\left(U^{2}\right)$ and that possibly uncontrolled corrections can only occur in $\mathcal{O}\left(U^{3}\right)$. Figures 4(b) and 4(c) show the current in the limit $\Gamma \rightarrow$ 0 for different values of $U / t$. One observes a clear $U^{2}$ dependence (dashed red line), which is well under control in our approach. This rules out the possibility that the negative current is an artifact of our method.

One can gain some understanding of the counterintuitive finding of a negative current by considering the local effective distribution function:

$$
n_{i}^{\mathrm{eff}}(\omega)=\frac{1}{2}-\frac{\operatorname{Im}\left[\sum_{i i}^{\mathrm{K}}(\omega)\right]}{4 \operatorname{Im}\left[\Sigma_{i i}^{\mathrm{ret}}(\omega)\right]}
$$

Due to the combined translation and energy-shift symmetry $n_{i}^{\text {eff }}(\omega)=n_{i+j}^{\text {eff }}(\omega+j E)$, we can restrict ourselves to $n^{\text {eff }}(\omega) \equiv n_{0}^{\text {eff }}(\omega)$. In equilibrium, our method recovers the dissipation-fluctuation theorem, and Eq. (3) reduces to the Fermi-Dirac distribution function. In nonequilibrium, we take $n^{\text {eff }}(\omega)$ as a proxy which subsums interaction and driving effects into a distribution function that is effectively imprinted on the different lattice sites. The results are summarized in Fig. 4(d) (we note that this effect cannot be captured by a mean-field treatment). For $U=0$, every site 
features an effective zero-temperature distribution function imprinted by the reservoirs. However, at finite $U$ this situation changes drastically. At small to moderate $U$ and for frequencies within the bandwidth (those relevant for the transport), the effective distribution function $n^{\text {eff }}(\omega)$ inverts its slope (at high frequencies, one recovers the usual Fermi-Dirac behavior). This signals the onset of an effective negative temperature, and it is well understood that this leads to particles flowing in the opposite, i.e., up-bias, direction [25-27].

Conclusion.-We have studied BKT transitions beyond thermal equilibrium using a novel renormalization-groupbased framework. Our approach keeps track of all microscopic details during the RG flow, which is necessary in nonequilibrium where an a priori separation of energy scales is ambiguous. We have applied this machinery to a prototypical model of spinless interacting fermions on a one-dimensional lattice coupled to electronic reservoirs and driven out of equilibrium by an electric field. The closed system is known to exhibit a BKT transition between a metallic and a CDW phase in the equilibrium limit. Our method reproduces this result and also yields quantitative agreement with a mean-field prediction for the phase boundary in limits where quantum fluctuations become irrelevant (e.g., for strong interactions). This shows that our approach is well suited to determine the nonequilibrium phase diagram for arbitrary parameters. We demonstrated an intricate, highly nonmonotonic dependence of the phase boundary on the strength of the electric field, leading to reenterance behavior. Moreover, we reported on the emergence of a steady state in (certain parameters regimes of) the open system where a current is flowing upstream against the drive of the electric field; this state is reminiscent of an equilibrium distribution with an effective negative temperature. Our study can be extended to plethora of questions straightaway such as spinful systems, spin-orbit coupling, topological systems, or systems in two dimensions. Another fruitful direction of future research concerns the question in which sense our results can be interpreted by first mapping the microscopic theory to a continuum field theory Sine-Gordon model, which is known to work in equilibrium. Our results can provide a microscopic testbed to determine under which conditions such a mapping is valid even in the continuously driven strongly nonequilibrium realm, i.e., beyond the cases of quantum quenches [18,19]. Our approach is also extendable to the case of finite driving frequency of the electric field, which will allow us to directly connect to experiments using laser excitations of solids.

D. M. K. acknowledges funding by the Deutsche Forschungsgemeinschaft (DFG, German Research Foundation) under Germany's Excellence StrategyCluster of Excellence Matter and Light for Quantum Computing (ML4Q) EXC 2004/1-390534769. We acknowledge support from the Max Planck-New York
City Center for Non-Equilibrium Quantum Phenomena. C. Ka. and C. Kl. acknowledge support by the Deutsche Forschungsgemeinschaft through the Emmy Noether program (Program No. KA 3360/2-1). C. Ka. acknowledges support by the Lower Saxony QUANOMET initiative.

[1] D. N. Basov, R. D. Averitt, and D. Hsieh, Nat. Mater. 16, 1077 (2017).

[2] S. Sachdev, Quantum Phase Transitions (Cambridge University Press, Cambridge, England, 2009).

[3] M. Budden, T. Gebert, M. Buzzi, G. Jotzu, E. Wang, T. Matsuyama, G. Meier, Y. Laplace, D. Pontiroli, M. Ricc, F. Schlawin, D. Jaksch, and A. Cavalleri, arXiv:2002.12835.

[4] N. D. Mermin and H. Wagner, Phys. Rev. Lett. 17, 1133 (1966).

[5] P. C. Hohenberg, Phys. Rev. 158, 383 (1967).

[6] N. Walldorf, D. M. Kennes, J. Paaske, and A. J. Millis, Phys. Rev. B 100, 121110(R) (2019).

[7] S. G. Jakobs, V. Meden, and H. Schoeller, Phys. Rev. Lett. 99, 150603 (2007).

[8] A. Mitra and A.J. Millis, Phys. Rev. B 77, 220404(R) (2008).

[9] D. M. Kennes, S. G. Jakobs, C. Karrasch, and V. Meden, Phys. Rev. B 85, 085113 (2012).

[10] H. Aoki, N. Tsuji, M. Eckstein, M. Kollar, T. Oka, and P. Werner, Rev. Mod. Phys. 86, 779 (2014).

[11] L. M. Sieberer, M. Buchhold, and S. Diehl, Rep. Prog. Phys. 79, 096001 (2016).

[12] A. K. Eissing, V. Meden, and D. M. Kennes, Phys. Rev. Lett. 116, 026801 (2016).

[13] D. M. Kennes, A. de la Torre, A. Ron, D. Hsieh, and A. J. Millis, Phys. Rev. Lett. 120, 127601 (2018).

[14] M. E. Fisher, Rev. Mod. Phys. 46, 597 (1974).

[15] K. G. Wilson, Rev. Mod. Phys. 47, 773 (1975).

[16] W. Metzner, M. Salmhofer, C. Honerkamp, V. Meden, and K. Schönhammer, Rev. Mod. Phys. 84, 299 (2012).

[17] P. Kopietz, L. Bartosch, and F. Schütz, Introduction to the Functional Renormalization Group, Lecture Notes in Physics (Springer-Verlag Berlin Heidelberg, Heidelberg, 2010).

[18] L. Mathey and A. Polkovnikov, Phys. Rev. A 81, 033605 (2010).

[19] L. Mathey, K. J. Günter, J. Dalibard, and A. Polkovnikov, Phys. Rev. A 95, 053630 (2017).

[20] J. M. Kosterlitz and D. J. Thouless, J. Phys. C 6, 1181 (1973).

[21] T. Giamarchi, Quantum Physics in One Dimension (Clarendon Press, Oxford, 2004), p. 2905.

[22] G. H. Wannier, Rev. Mod. Phys. 34, 645 (1962).

[23] E. van Nieuwenburg, Y. Baum, and G. Refael, Proc. Natl. Acad. Sci. U.S.A. 116, 9269 (2019).

[24] M. Schulz, C. A. Hooley, R. Moessner, and F. Pollmann, Phys. Rev. Lett. 122, 040606 (2019).

[25] N. F. Ramsey, Phys. Rev. 103, 20 (1956).

[26] A. P. Mosk, Phys. Rev. Lett. 95, 040403 (2005).

[27] S. Braun, J. P. Ronzheimer, M. Schreiber, S. S. Hodgman, T. Rom, I. Bloch, and U. Schneider, Science 339, 52 (2013). 
[28] A. V. Andreev, I. L. Aleiner, and A. J. Millis, Phys. Rev. Lett. 91, 056803 (2003).

[29] I. A. Dmitriev, M. G. Vavilov, I. L. Aleiner, A. D. Mirlin, and D. G. Polyakov, Phys. Rev. B 71, 115316 (2005).

[30] A. Kamenev and A. Levchenko, Adv. Phys. 58, 197 (2009).

[31] R. Gezzi, T. Pruschke, and V. Meden, Phys. Rev. B 75, 045324 (2007).

[32] D. M. Kennes and V. Meden, Phys. Rev. B 88, 165131 (2013).

[33] C. Karrasch, R. Hedden, R. Peters, T. Pruschke, K. Schnhammer, and V. Meden, J. Phys. Condens. Matter 20, 345205 (2008).

[34] S. G. Jakobs, M. Pletyukhov, and H. Schoeller, Phys. Rev. B 81, 195109 (2010).

[35] C. Kloeckner, D. M. Kennes, and C. Karrasch, New J. Phys. 22, 083039 (2020).
[36] L. Markhof, B. Sbierski, V. Meden, and C. Karrasch, Phys. Rev. B 97, 235126 (2018).

[37] Y. Taguchi, T. Matsumoto, and Y. Tokura, Phys. Rev. B 62 , 7015 (2000).

[38] E. Boulat, H. Saleur, and P. Schmitteckert, Phys. Rev. Lett. 101, 140601 (2008)

[39] T. S. Inada, I. Terasaki, H. Mori, and T. Mori, Phys. Rev. B 79, 165102 (2009).

[40] T. Mori, T. Ozawa, Y. Bando, T. Kawamoto, S. Niizeki, H. Mori, and I. Terasaki, Phys. Rev. B 79, 115108 (2009).

[41] See the Supplemental Material at http://link.aps.org/ supplemental/10.1103/PhysRevLett.125.147601 for details on the numerics and its convergence. 